\title{
Uncertainties on propagation parameters: impact on the interpretation of the positron fraction
}

\section{Mathieu Boudaud ${ }^{a}$, Sandy Aupetit ${ }^{a}$, Sami Caroff ${ }^{b}$, Antje Putze ${ }^{a}$, Geneviève Bélanger $^{a}$, Yoann Genolini ${ }^{* a}$, Corine Goy ${ }^{b}$, Vincent Poireau ${ }^{b}$, Vivian Poulin ${ }^{a}$, Sylvie Rosier $^{b}$, Pierre Salati $^{a}$, Li Tao $^{b}$, and Manuela Vecchi ${ }^{c}$}

${ }^{a}$ LAPTh, Université Savoie Mont Blanc \& CNRS, 9 Chemin de Bellevue, B.P.110 Annecy-le-Vieux, F-74941, France

${ }^{b}$ LAPP, Université Savoie Mont Blanc \& CNRS, 9 Chemin de Bellevue, B.P.110 Annecy-le-Vieux, F-74941, France

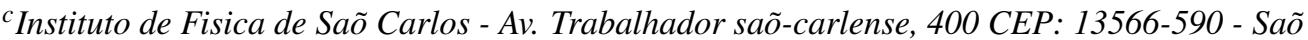

Carlos (SP), Brazil

E-mail: yoann.genolini@lapth.cnrs.fr

\begin{abstract}
The positron fraction in cosmic rays has recently been measured with improved accuracy up to $500 \mathrm{GeV}$, and it was found to be a steadily increasing function of energy above $\sim 10 \mathrm{GeV}$. This behaviour contrasts with standard astrophysical mechanisms, in which positrons are secondary particles, produced in the interactions of primary cosmic rays during their propagation in the interstellar medium. The observed anomaly in the positron fraction triggered a lot of excitement, as it could be interpreted as an indirect signature of the presence of dark matter species in the Galaxy, the so-called weakly interacting massive particles (WIMPs). Alternatively, it could be produced by nearby sources, such as pulsars. These hypotheses are probed in [1], in light of the latest AMS-02 positron fraction measurements. The cosmic ray positron transport in the Galaxy is described using a semi-analytic two-zone model. For consistency, the secondary and primary components of the positron flux are calculated together with the same propagation model. We show that the results inferred for both hypotheses crucially depend on the propagation parameters, estimated with the Boron-to-Carbon ratio. Their uncertainties turn out to be very significant, and overshadow even the statical errors from the positron data.
\end{abstract}

The 34th International Cosmic Ray Conference,

30 July- 6 August, 2015

The Hague, The Netherlands

\footnotetext{
* Speaker.
} 


\section{Introduction}

The cosmic ray positron flux at the Earth exhibits above $10 \mathrm{GeV}$ an excess with respect to the astrophysical background produced by the interactions of high-energy protons and helium nuclei with the interstellar medium (ISM) [2, 3, 4]. Recently, the Alpha Magnetic Spectrometer (AMS02) collaboration has published [5] an update on the positron fraction based on high statistics with measurements extending up to $500 \mathrm{GeV}$. In [1] we studied the constraints on an additional contribution of DM or a single pulsar to explain the positron fraction measured above $10 \mathrm{GeV}$. These constraints have been obtained by modelling the expected positron flux with the cosmic ray diffusion benchmark model MED defined in [6]. However, the transport mechanisms of Galactic cosmic rays are still poorly understood. The uncertainties on cosmic ray transport parameters are not negligible and have a major impact on searches for new physics. To take these uncertainties into account and to study their effect on modelling the positron fraction with an additional contribution, we use a set of 1623 combinations of the transport parameters $\left\{\delta, K_{0}, L, V_{c}, V_{A}\right\}$. These parameter sets result from a secondary-to-primary ratio analysis [7] where 26 data points of the boron-tocarbon $(\mathrm{B} / \mathrm{C})$ ratio were fitted over the energy range from 0.1 to $35 \mathrm{GeV} / \mathrm{n}$ leading to a $\chi^{2}$ less than 40. The advantage of choosing this study over more recent studies $[8,9,10]$ are the wider and more conservative ranges of the transport parameters. In addition, the benchmark models MIN, MED, and MAX of[6], widely used in the DM literature, are based on the parameters found in [7].

In the following we extrapolate these models to higher energies. We furthermore marginalise over $V_{c}$ and $V_{A}$ since the reaccelerating and convection processes are negligible at higher energies and are not taken into account in our positron flux calculation. Finally, we only show the $\chi \chi \rightarrow b \bar{b}$ channel and the pulsar J1745-3040 as an example to highlight the correlations between the transport parameters and the parameters necessary to model the additional exotic contribution to the positron fraction at higher energies.
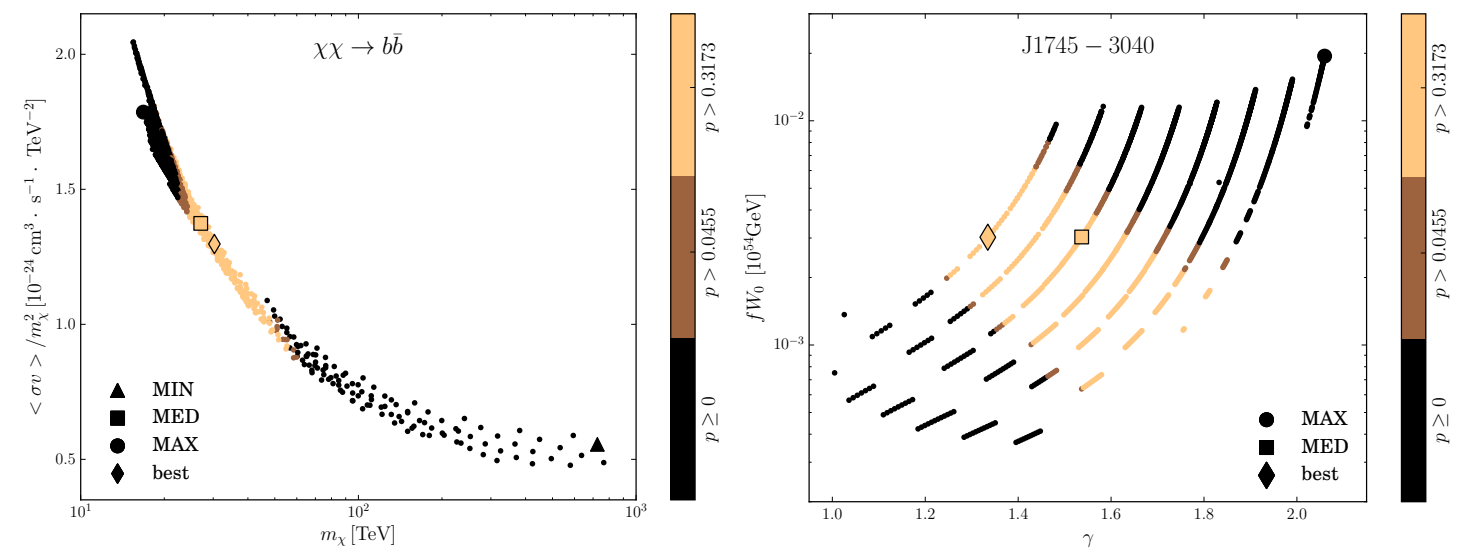

Figure 1: $p$-value distributions of the 1623 transport parameter sets for the DM $\left\{m_{\chi},\langle\sigma v\rangle / m_{\chi}^{2}\right\}$ (left plot) and the pulsar $\{\gamma, f W 0\}$ (right plot) parameters. The colour coding represents the increasing $p$-value from darker to lighter colours. The benchmark models MIN, MED, and MAX are represented with a triangle, square, and circle symbol, respectively. In addition, the best transport parameter set is highlighted with a diamond symbol. 


\section{Which transport parameters give a good fit?}

For each set of transport parameters, we fit the positron fraction to find the best combination of $\left\{\langle\sigma v\rangle, m_{\chi}\right\}$ or $\left\{f W_{0}, \gamma\right\}$ for the DM and pulsar contributions, respectively. We calculate the $p$-value to determine for which transport parameter set the modelled sum of secondary and exotic contributions reproduce well the positron fraction measured by AMS-02. In Fig. 1, the $p$-value distributions of the 1623 transport parameter sets for the DM $\left\{m_{\chi},\langle\sigma v\rangle / m_{\chi}^{2}\right\}$ (left plot) and the pulsar $\{\gamma, f W 0\}$ (right plot) parameters are shown. The colour coding represents the increasing $p$-value from darker to lighter colours, which is binned into the three defined $p$-value ranges. The higher the $p$-value, the better the modelled positron fraction reproduces the experimental data. When $p \leq 0.0455$ the model is excluded for the final results.

The benchmark models MIN, MED, and MAX are represented with a triangle, square, and circle symbol, respectively. In addition, the best transport parameter set is highlighted with a diamond symbol. For the pulsar J1745-3040 some transport models resulting in a very low and unphysical $\gamma$ values were excluded from the analysis, including the benchmark model MIN. The criterion of goodness-of-fit defined above reduces the number of transport parameter sets considered from 1623 to a few hundred. In general, the benchmark models MIN (triangle) and MAX (filled circle) are disfavoured by the experimental data. We observe on these figures that the transport parameters are strongly correlated with the DM and pulsar parameters. We discuss these correlations in the following section.

\section{Correlations between parameters}
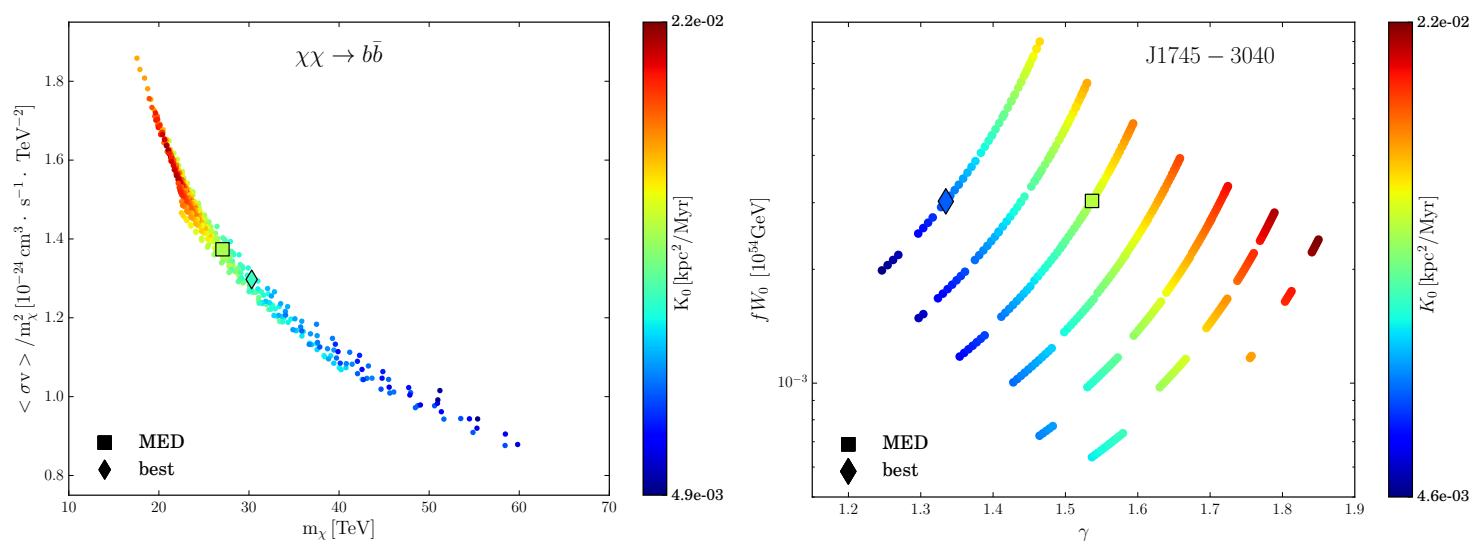

Figure 2: Surviving transport parameter sets' $K_{0}$ distributions for the $\operatorname{DM}\left\{m_{\chi},\langle\sigma v\rangle / m_{\chi}^{2}\right\}$ (left plot) and the pulsar $\left\{\gamma, f W_{0}\right\}$ (right plot) parameters. The colour coding represents the increasing $K_{0}$ value from blue to red. The benchmark model MED is represented with a square. In addition, the best transport parameter set is highlighted with a diamond symbol.

Figures 2 and 3 show the best fit for each transport parameter set (coloured dot) in the $\langle\sigma v\rangle / m_{\chi}^{2}$ $-m_{\chi}$ (left plots) and $\gamma-f W_{0}$ (right plots) planes, representing the correlations between the transport parameters and DM or pulsar parameters. Plotting $\langle\sigma v\rangle / m_{\chi}^{2}$ instead of $\langle\sigma v\rangle$ allows us to clearly see the correlations. The colour indicates the value of a given transport parameter $\left(K_{0}\right.$ in Fig. $2, \delta$ in 

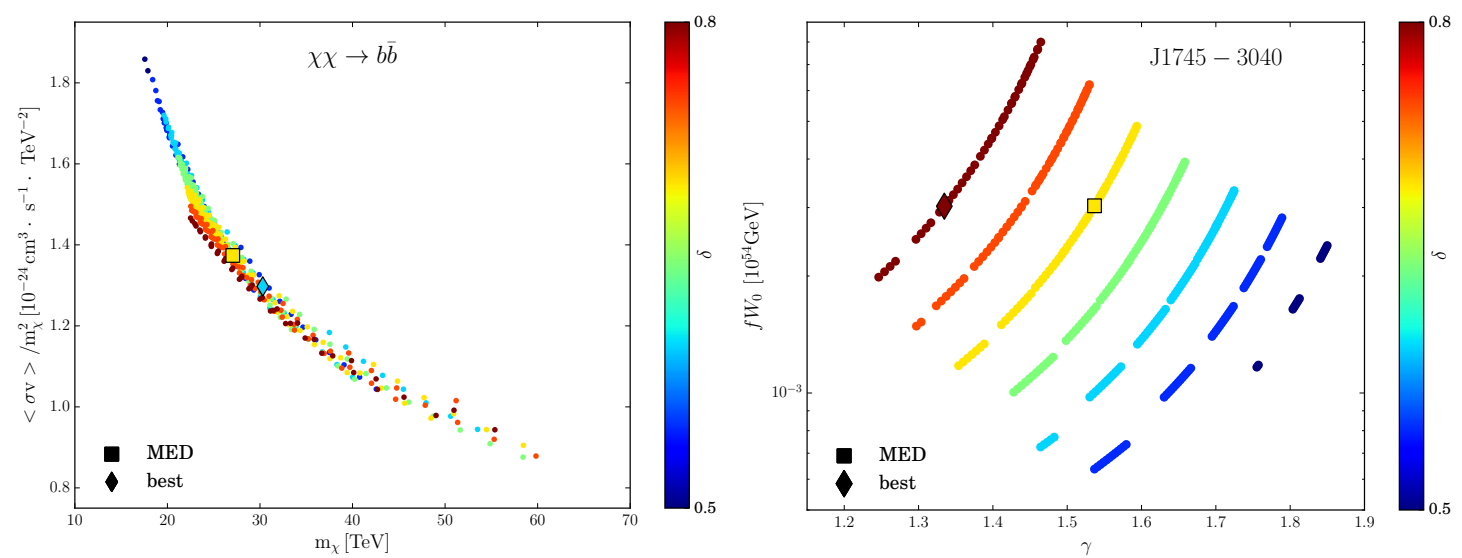

Figure 3: Surviving transport parameter sets' $\delta$ distributions for the $\operatorname{DM}\left\{m_{\chi},\langle\sigma v\rangle / m_{\chi}^{2}\right\}$ (left plot) and the pulsar $\left\{\gamma, f W_{0}\right\}$ (right plot) parameters. The colour coding represents the increasing $\delta$ value from blue to red. The benchmark model MED is represented with a square. In addition, the best transport parameter set is highlighted with a diamond symbol.

Fig. 3) from lower (blue) to higher (red) values. One can clearly see a strong correlation between the transport and DM or pulsar parameters showing a huge impact on the best-fit values for the considered free parameters.

The main correlations are due to the normalisation and the shape of the fluxes. Indeed, $\langle\sigma v\rangle / m_{\chi}^{2}$ and $f W_{0}$ are related to the number of positrons injected in the $\mathrm{MH}$, whereas the amount of produced secondary particles is inversely proportional to the diffusion length $\lambda_{D}$ (see Eq. 7 in [1]) and is hence negatively correlated with $K_{0}$. If enough secondary particles are created, we need fewer particles through DM or pulsar injection and vice-versa. For the DM candidate, $\langle\sigma v\rangle / m_{\chi}^{2}$ increases with $K_{0}$ as shown in the left panel of Fig. 2. In the case of the J1745-3040 pulsar, that trend is reinforced by the fact that given the small distance of the source, the positron flux scales as $\lambda_{D}^{-3} \propto K_{0}^{-3 / 2}$. We observe hence a strong positive correlation between $f W_{0}$ and $K_{0}$ in the right panel of Fig. 2. The spectral index of the diffusion coefficient $\delta$ modifies the high energy shape of the secondary positron flux. A lower (higher) value of $\delta<0.5(\delta>0.5)$ has a harder (softer) spectrum and therefore allows for a DM induced contribution at smaller (higher) DM masses $m_{\chi}$. This trend is clearly visible in the left panel of Fig. 3. The correlation between $\langle\sigma v\rangle / m_{\chi}^{2}$ and $m_{\chi}$ is due to the form of the DM spectrum and varies from channel to channel. For pulsars, the primary positron flux behaves as $f W_{0} / E^{(\gamma+3 \delta / 2)}$. This scaling accounts for the positive (negative) correlation between $f W_{0}(\gamma)$ and the spectral index $\delta$ observed in the right panel of Fig. 3 .

In each of the above figures, the best transport parameter set (highest $p$-value) is shown (diamond symbol) for the $\chi \chi \rightarrow b \bar{b}$ channel and the pulsar J1745-3040. In the same way, we also extract the best set of parameters for the other studied DM annihilation channels and pulsars. The results are summarised in Tab. 4 and 5 of [1]. In each case, independent of the primary positron source, we can find a set of parameters that better describes the experimental data than the benchmark model MED. Moreover, in the framework of our analysis, the experimental data favour small halo sizes $(L \lesssim 3.5 \mathrm{kpc}$ ). Eventually, taking the uncertainties into account of the propagation parameters does not change the discrepancy between the AMS-02 data and the modelled positron 
fraction, neither for the electron DM annihilation channel nor for the Monogem and Vela pulsars.

\section{Comparison of systematic and statistical uncertainties}
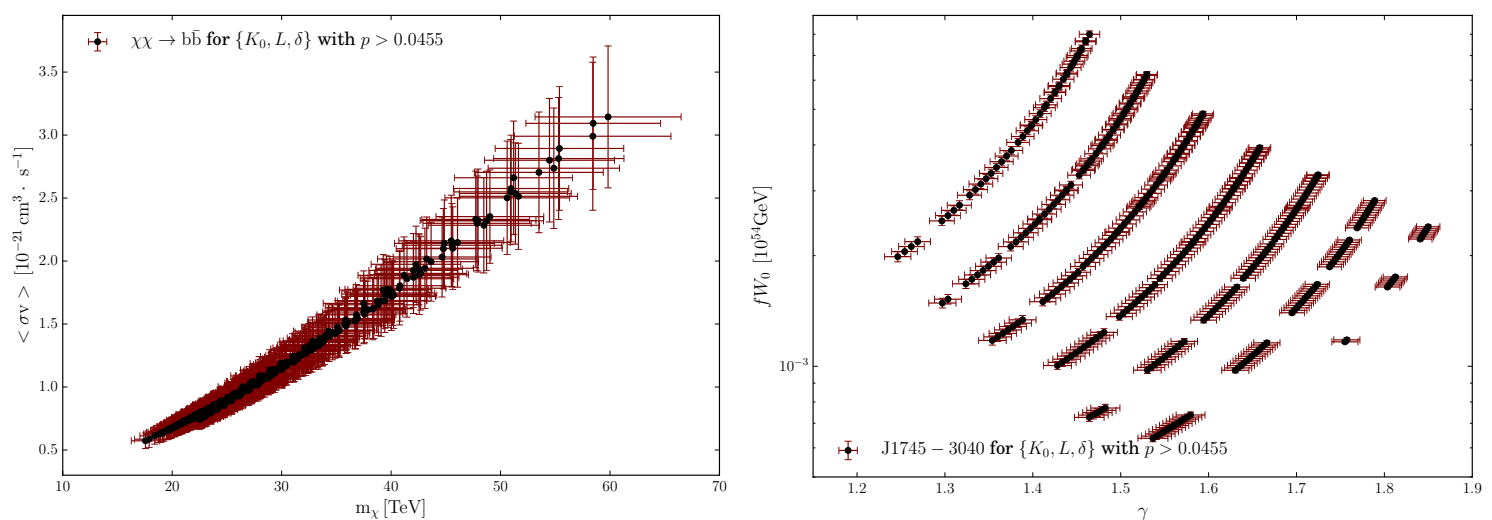

Figure 4: Best-fit values of the surviving transport parameter sets for the DM $\left\{m_{\chi},\langle\sigma v\rangle\right\}$ (left plot) and the pulsar $\{\gamma, f W 0\}$ (right plot) parameters. The error bars represent the errors on the fit parameter resulting from the statistical uncertainty on the experimental data.

The goodness-of-fit criterion ( $p>0.0455)$ allows us to select the parameter sets that describe the experimental data reasonably well. The spread of these parameter sets shown in Fig. 4 (black dots) in the $\langle\sigma v\rangle-m_{\chi}$ and $\gamma-f W_{0}$ planes therefore represents the systematic uncertainty of the determination of $\langle\sigma v\rangle$ and $m_{\chi}$ as well as $\gamma$ and $f W_{0}$. Compared to the statistical uncertainties of these parameters because of the errors of the experimental data (red crosses), the systematic uncertainties dominate completely their determination. A perfect knowledge of the distance and age of the pulsar is assumed: in reality, this is not the case and would lead to larger uncertainties. The inclusion of the uncertainties on the pulsar distance is beyond the scope of this analysis and will be considered in a follow-up study. To better estimate the transport parameters and reduce their impact on the study of an additional contribution to the positron fraction, more precise measurements of secondary-to-primary ratios over a large energy range are needed.

\section{How can the positron fraction constrain the diffusive halo size?}

The $\mathrm{B} / \mathrm{C}$ ratio is sensitive to the matter density in the Galactic disc, which is related to $L / K_{0}$. This degeneracy can be broken by an observable, which is sensitive to only one of the two parameters. In general, one uses radioactive secondary-to-stable secondary ratios, such as ${ }^{10} \mathrm{Be} /{ }^{9} \mathrm{Be}$. The radioactive secondaries decay before they can reach the edge of the Galaxy and escape. Their modelling is hence independent of the Galactic diffusive halo size $L$. Because of a lack of precise measurements over a sufficient large energy range up to now, the halo size is still not estimated well. Recently, [11] demonstrated that low-energetic secondary positrons can directly constrain diffusion models with small haloes and large spectral indices due to very high energy losses and hence small diffusion lengths. Besides the availability of very precise positron data over a large energy range, this method is less sensitive to the modelling of the local interstellar medium compared 

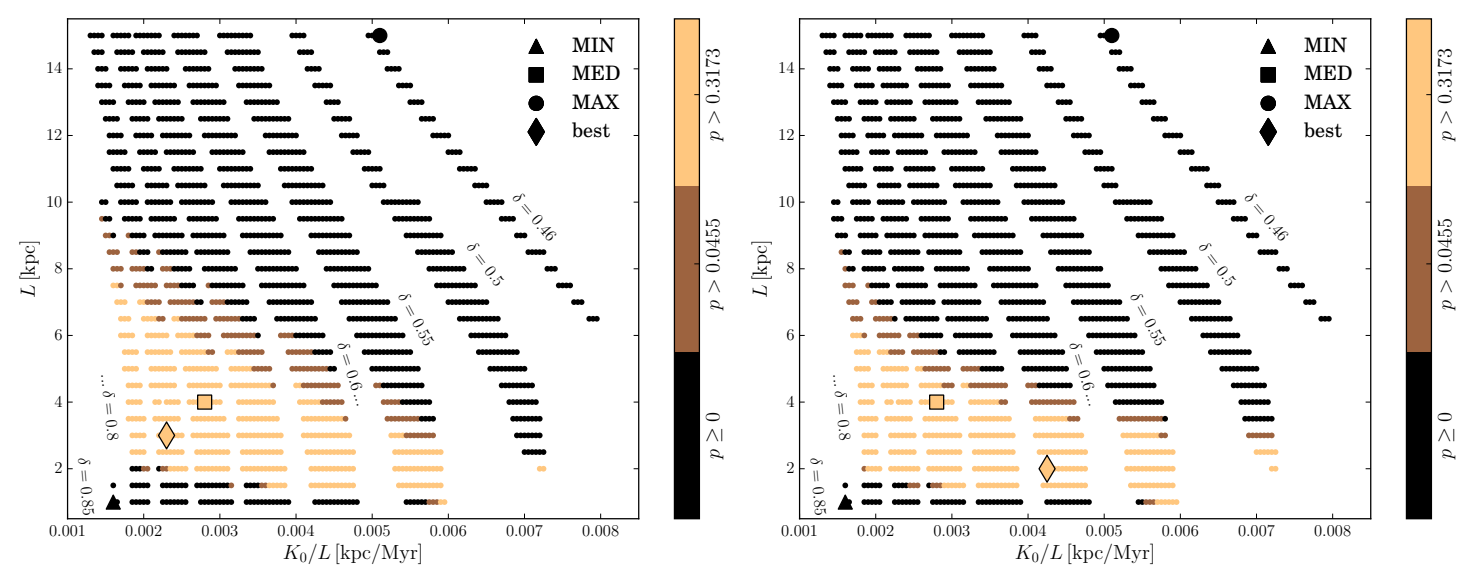

Figure 5: For each transport parameter set, represented in the $K_{0} / L-L$ plane, the best $p$-value distributions of all the 12 channels (five pulsars) are shown in the left (right) plot. The colour coding represents the increasing $p$-value from darker to lighter colours. The benchmark models MIN, MED, and MAX are represented with a triangle, square, and circle symbol, respectively. In addition, the best transport parameter set is highlighted with a diamond symbol.

to the standard approach. In this study, only a secondary positron spectrum was used. We propose to extend the analysis by considering here an additional contribution to the positron fraction from DM annihilation or pulsars as well as taking their different spectral shapes into account.

Figure 5 shows the 1623 transport parameter sets in the $\left(K_{0} / L, L\right)$ plane. As before, we divide the sets into three different bins of $p$-values obtained by a fit with a contribution from either a given DM channel or a single pulsar. For each transport parameter set, we choose the best $p$-value from all the 12 (five) channels for the DM (single pulsar) contribution considered in the analysis of [1]. In both cases, very small $(L \lesssim 2 \mathrm{kpc})$ and very big halo sizes $(L \gtrsim 7 \mathrm{kpc})$ as well as small diffusion slopes $(\delta \lesssim 0.5)$ are disfavoured by the experimental data due to the different spectral features at high energies of the additional contribution. In our analysis, the benchmark models MIN and MAX are largely disfavoured by the experimental data. However these constraints are model dependent since they are sensitive to the shape of the additional contribution.

\section{Conclusion}

Whatever DM or pulsars hypothesis is favoured to explain the rise of the positron fraction, the conclusions drawn in [1] assume a given set of cosmic ray transport parameters derived from the boron-to-carbon analysis of [7] and dubbed MED in [6]. However, the transport mechanisms of charged cosmic rays are still poorly understood, necessitating the inclusion of their uncertainties in the studies of the rise of the positron fraction. In this work, we use 1623 different transport parameter sets, all in good agreement with nuclear measurements. We observe that the error arising from the propagation uncertainties is much larger than the statistical uncertainty on the fitted parameters. In conclusion, the ignorance of the exact transport parameter values is the main limitation of such analyses. Henceforth, the study of cosmic ray propagation should be the main focus of future experiments. 


\section{References}

[1] M. Boudaud, S. Aupetit, S. Caroff, A. Putze, G. Belanger, Y. Genolini, C. Goy, V. Poireau, V. Poulin, S. Rosier, P. Salati, L. Tao, and M. Vecchi. A new look at the cosmic ray positron fraction. A\&A, 575:A67, March 2015.

[2] Barwick et al. and HEAT Collaboration. Measurements of the Cosmic-Ray Positron Fraction from 1 to $50 \mathrm{GeV}$. APJL, 482:L191-L194, June 1997.

[3] DuVernois et al. Cosmic-Ray Electrons and Positrons from 1 to $100 \mathrm{GeV}$ : Measurements with HEAT and Their Interpretation. APJ, 559:296-303, September 2001.

[4] Beatty et al. New Measurement of the Cosmic-Ray Positron Fraction from 5 to $15 \mathrm{GeV}$. Physical Review Letters, 93(24):241102, December 2004.

[5] L. Accardo et al. High Statistics Measurement of the Positron Fraction in Primary Cosmic Rays of 0.5-500 GeV with the Alpha Magnetic Spectrometer on the International Space Station. Phys.Rev.Lett., 113:121101, 2014.

[6] F. Donato, N. Fornengo, D. Maurin, P. Salati, and R. Taillet. Antiprotons in cosmic rays from neutralino annihilation. PRD, 69(6):063501, March 2004.

[7] D. Maurin, F. Donato, R. Taillet, and P. Salati. Cosmic Rays below Z=30 in a Diffusion Model: New Constraints on Propagation Parameters. APJ, 555:585-596, July 2001.

[8] A. Putze, L. Derome, and D. Maurin. A Markov Chain Monte Carlo technique to sample transport and source parameters of Galactic cosmic rays. II. Results for the diffusion model combining $\mathrm{B} / \mathrm{C}$ and radioactive nuclei. $A \& A, 516:$ A66, June 2010.

[9] B. Coste, L. Derome, D. Maurin, and A. Putze. Constraining Galactic cosmic-ray parameters with Z $\leq 2$ nuclei. $A \& A$, 539:A88, March 2012.

[10] R. Trotta, G. Jóhannesson, I. V. Moskalenko, T. A. Porter, R. Ruiz de Austri, and A. W. Strong. Constraints on Cosmic-ray Propagation Models from A Global Bayesian Analysis. APJ, 729:106, March 2011.

[11] J. Lavalle, D. Maurin, and A. Putze. Direct constraints on diffusion models from cosmic-ray positron data: Excluding the minimal model for dark matter searches. PRD, 90(8):081301, October 2014. 